\title{
ATENUACIÓN: ALGUNAS CLAVES METODOLÓGICAS PARA SU ANÁLISIS ${ }^{1}$
}

\author{
MITIGATION: SOME METHODOLOGICAL KEYS FOR ITS ANALYSIS
}

\section{Cristina Villalba Ibáñez}

Universitat de València/Universitat Jaume I

Resumen

El estudio de la atenuación lingüística, en tanto fenómeno pragmático, necesita criterios consensuados de identificación que ayuden al analista en sus investigaciones y facilite el desarrollo de estudios contrastivos. En esta línea, este artículo se propone reflexionar acerca del fenómeno de la atenuación y, más concretamente, sobre los criterios que la bibliografía ha empleado para su reconocimiento. Dichos criterios podrían resumirse grosso modo en la vaguedad del contenido semántico, la mitigación de la fuerza ilocutiva, la desfocalización del papel de los participantes en la interacción y el reconocimiento de la función o finalidad de la atenuación. Además de estos criterios, se ofrecen tres pruebas que pueden emplearse para validar el análisis: la prueba de la ausencia, la prueba de la conmutación y la prueba de la solidaridad. Estas pruebas, junto con un estudio pormenorizado del contexto interactivo general (el género discursivo) y el contexto interactivo concreto en el que aparece el mecanismo atenuante pueden resultar claves para reforzar los criterios anteriormente señalados y facilitar el reconocimiento de la atenuación.

\begin{abstract}
The study of linguistic mitigation, understood as a pragmatic phenomenon, requires consensus-based criteria in order to help analysts in their investigations and facilitate the development of contrastive studies. In this sense, this article aims to reflect on the phenomenon of attenuation and, more precisely, on the criteria presented in the literature to identify it. These criteria could be summed up in the vagueness of the semantic content, the mitigation of illocutive force, the defocalization of the participants in the interaction and the recognition of the function or purpose of mitigation. In addition to these criteria, we will offer three proofs that can be used to validate the analysis: the absence proof, the commutation proof and the solidarity proof. These proofs, as well as the general interactive context (discoursive genre) and the concrete interactive context can be paramount to strengthen the criteria previously presented and to facilitate the recognition of mitigation.
\end{abstract}

KEY WORDS: pragmatics, mitigation, methodology, analysis criteria

PALABRAS CLAVE: pragmática, atenuación, metodología, criterios de análisis

\footnotetext{
${ }^{1}$ Esta investigación ha sido posible gracias a financiación de dos proyectos de investigación: "La atenuación pragmática en el español hablado: su variación diafásica y diatópica" (Ministerio de Economía y Competitividad de España, ref. FFI201340905-P) y Es.VaG.Atenuación ("La atenuación pragmática en su variación genérica: géneros discursivos escritos y orales en el español de España y América"; Ministerio de Economía y Competitividad de España, ref. FFI2016-75249-P).
} 


\section{INTRODUCCIÓN}

Tradicionalmente, los estudios sobre atenuación han proporcionado catálogos de formas para ayudar a identificar este fenómeno. En algunos casos, las investigaciones se centran en mecanismos concretos, como los aproximativos (Lakoff 1972), los verbos parentéticos (Lysvåg 1975), los deícticos (Haverkate, 1992) o los diminutivos (Boretti de Macchia y Ferrer de Gregoret, 1984), por citar algunos ejemplos. En otros casos, se prefiere proporcionar catálogos más generales que sirvan de guía al analista (Albelda, 2010b; Albelda, Briz, Cestero, Kotwica, y Villalba, 2014; Albelda y Cestero, 2011, 2012; Briz, 2001; Caffi, 2007; Holmes, 1984a; Meyer-Hermann, 1988 y Schneider, 2013).

La existencia de catálogos de mecanismos potencialmente atenuantes puede ser una herramienta útil para el reconocimiento de la atenuación, puesto que, en cierta medida, ayuda a orientar la mirada del analista. La elaboración de estos catálogos, sobre todo en los primeros trabajos sobre atenuación, se basó en la intuición lingüística del hablante o de informantes. Posteriormente, la elaboración de estos catálogos se ha ido apoyando en el análisis de corpus, de manera que se constata la posible relación entre una forma lingüística y la atenuación.

Ahora bien, el uso de estos catálogos para rastrear los casos de atenuación entraña otro problema, ya que una misma forma lingüística puede desarrollar (o no) un valor atenuante según su uso concreto (Albelda 2010:48). En consecuencia, además de la identificación de una forma candidata a desarrollar atenuación, hay que combinar otros criterios de análisis que nos permitan confirmar el valor atenuante. Esto no es una tarea sencilla debido, precisamente, a la dependencia contextual de la atenuación. En esta línea, puede hacerse difícil para el analista identificar con claridad la intención del que emite el enunciado en algunos casos. Más allá de estas limitaciones, que están íntimamente ligadas a la pragmática, nuestra propuesta en este trabajo es reflexionar sobre las características y los criterios que se han ofrecido para identificar la atenuación y aportar una serie de pruebas que, junto con un análisis profundo del contexto, pueden contribuir a facilitar el análisis de este fenómeno.

\section{A QUÉ AFECTA LA ATENUACIÓN}

En los últimos años, la atenuación se ha convertido en un tema de investigación popular dentro de la comunidad científica, como muestra el gran número de trabajos, congresos y proyectos dedicados de manera directa o indirecta a su estudio². Originalmente, el estudio de la atenuación surgió en relación con el componente semántico. Lakoff (1972) recuperando algunas aportaciones de Zadeh (1965), reflexiona sobre aquellos elementos lingüísticos cuyo valor impreciso, vago o difuso (fuzzy en términos del autor) pueden afectar a los valores de verdad de un enunciado. Esto le lleva a elaborar un catálogo de recursos como más o menos o de alguna manera ${ }^{3}$ que impiden que el elemento afectado por este modificador se identifique claramente con la categoría a la que pertenece, como sucede en el ejemplo (1).

\footnotetext{
${ }^{2}$ La página del proyecto Es.Var.Atenuación y Es.Vag.Atenuación recoge la bibliografía y algunos de los eventos más importantes relacionados con la atenuación (www.esvaratenuacion.es).

${ }^{3}$ La traducción es nuestra. Para ver con más detalle la lista, confróntese con Lakoff (1972:472). 
(1) A: o sea por otros medios sí que / la magia te dice pues haz esto o lo otro y lo conseguirás/ aquí lo único que te dice es bueno [esto puedes conseguirlo $\uparrow$ ]

B:

[bueno $\downarrow$ pero de alguna for-] de alguna forma te apoya $\downarrow$ te

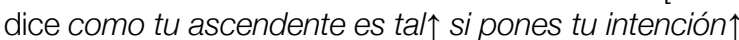

(Briz et al. 2002:356)

¿La hablante B afirma que la magia apoya a las personas? La respuesta sería sí y no: de acuerdo con su enunciado, las apoya, pero no de una manera suficientemente prototípica como para decir abiertamente que las apoya. Este conflicto entre los valores de verdad de la proposición sirven de base para que se desarrollen estudios posteriores en los que no solo se tenga en cuenta el plano semántico, sino que se aborde su estudio desde un punto de vista pragmático que presta especial atención a la modulación de la fuerza ilocutiva (Albelda, 2010a; Bazzanella, 2002; Bazzanella, Caffi, y Sbisà, 1991; Caffi, 2007; Holmes, 1984b; Holmlander, 2006; Sbisà, 2001; Schneider, 2013; Thaler, 2012).

Estos dos planos, el semántico y el pragmático, pueden servir de ayuda al analista para llevar a cabo su investigación. Por una parte, se puede atenuar lo dicho mediante la difusión o la vaguedad del significado y, a partir de ahí, afectar a la fuerza ilocutiva (Albelda y Cestero, 2011; Briz, 1995; Caffi, 2007; Holmes, 1984b; Meyer-Hermann, 1988; Mihatsch, 2018; Sbisà, 2001). Es lo que sucede en un enunciado como (2a), donde el impacto en la fuerza ilocutiva nos permite identificar su valor atenuante. En este caso, la hablante, que está en casa de una amiga, le dice que tiene frío de una manera más indirecta mediante el uso del diminutivo. Esto contrasta con (2b), donde la falta de incidencia del diminutivo en el plano de la enunciación (tiene, en este caso, un valor afectivo) nos permite descartar su valor atenuante.

(2) a. Hace fresquito. ¿Me puedes dejar una chaqueta?

b. Me he tomado un zumo bien fresquito.

En relación con estos ejemplos, parece plausible que la minimización de la fuerza ilocutiva constituya un criterio para determinar si estamos ante un mecanismo de atenuación. Un ejemplo de ello es lo que sucede en enunciados como (3), extraído de un juicio oral.

(3) A: SIN EMBARGO// en frente/ tenemos la declaración// de don Daniel de la Mora Sáez que aquí se enfada $\uparrow$ que aquí se altera $\uparrow$ que aquí se cabrea / que incluso ENTIENDE que cuando un agente de la policía local/ debidamente uniformado como va esta mañana el inspector/ le PIDE la documentación y le dice que le va a denunciar $\uparrow$ denuncia administrativa $\downarrow$ ahora estamos en el ámbito penal pero era una simple denuncia / administrativa LE DICE que le va a denunciar ÉL entiende que le está vacilando $\downarrow$ lo ha dicho varias veces/ no solo entiende que le está vacilando sino que en un momento/ en el fragor de la discusión que mantiene ÉL exclusivamente porque ni el inspector ni el agente le levantan la voz/ en el fragor de esa discusión que solo él mantiene hasta se atreve/ a quitarse la camiseta/ eeh PROVOCANDO al agente $\downarrow$ provocando al inspector para que/ eh/ NO VALE PARA NADA entendemos que no vale para nada la declaración de don Daniel de la Mora

(Villalba, 2017:99)

En (3) se muestra cómo el papel de unuio de los participantes en la interacción (en este caso, el hablante) se desdibuja. Como consecuencia de esto, el acto de habla se formula de manera más indirecta, al reducirse el compromiso del hablante con la aserción. Este ejemplo es especialmente ilustrativo porque permite conocer la formulación lingüística más comprometida en términos epistémicos, que es la que aparece en primer lugar, y el elemento atenuador entendemos. Este verbo y, sobre todo, su formulación mediante la primera persona del plural (en lugar del singular) le sirven al emisor para distanciarse del 
enunciado, presentar la aserción de manera indirecta y, de esta manera, mitigar la fuerza ilocutiva.

\section{IDENTIFICACIÓN DE LA MOTIVACIÓN: FUNCIONES}

En el epígrafe anterior se ha visto que la atenuación es un fenómeno pragmático que se relaciona con la reducción, mitigación o minoración de la fuerza ilocutiva, ya sea directamente, a través del plano semántico o a partir de la desfocalización de los participantes en la interacción (Briz y Albelda, 2013; Caffi, 1999). Es el momento de preguntarse ahora por los motivos que llevan a un hablante a emplear mecanismos atenuantes.

El uso de la atenuación se relaciona con la eficiencia comunicativa, es decir, podremos hablar propiamente de atenuación cuando sea posible identificar el motivo por el que se elige un mecanismo atenuante. Si volvemos al ejemplo (3), uno de los elementos que nos permite determinar que es atenuante es valorar si existe algún motivo para utilizar el verbo entender y el uso de la primera persona del plural. Solo de esta manera, el hablante crea la ilusión de que su enunciado es objetivo, se presenta como una persona razonable libre de intereses particulares y facilita la aceptación de la tesis propuesta (Hyland, 1996; Villalba, 2018).

A la hora de explicar para qué usamos de la atenuación, frecuentemente se recurre a las obligaciones o los compromisos que establecen los participantes con lo dicho, en línea con lo propuesto por Meyer-Hermann (1988). Hablar de obligaciones o compromisos permite evitar utilizar el término imagen en la definición de atenuación (véase como ejemplo la definición de Briz y Albelda 2013:292). Esto puede deberse a dos razones.

En primer lugar, puede responder al deseo de definir el fenómeno en términos exclusivamente lingüísticos y considerar secundaria la esfera social (Albelda, 2018:352). En segundo lugar, no recurrir a la imagen trata de evitar que atenuación y cortesía se entiendan como sinónimos (Briz, 2012; Hernández Flores, 2013).

De todos modos, lo cierto es que a la hora de juzgar la presencia o ausencia de atenuación, se ha revelado como un factor relevante identificar la manera en que se gestionan las imágenes de los participantes (Cabedo Nebot, 2018a:1018). Esto hace pensar que atenuación e imagen están íntimamente relacionadas (Albelda, 2016) y que, muy probablemente, deba partirse de un concepto de imagen más amplio que integre las obligaciones y compromisos de los participantes.

Siguiendo la propuesta de Briz (2001, 2006, 2011, 2012), la atenuación puede cumplir tres macrofunciones (Villalba, 2016, 2017): autoprotección, prevención o reparación de la imagen. Antes de explicar a qué se refieren estas macrofunciones, es necesario dedicar unas líneas a explicar qué entendemos por macrofunción. Los trabajos más recientes que han triangulado los conceptos de imagen, género y atenuación (Albelda, 2018; Figueras, 2018) han mostrado que el género constriñe la manera en que se gestionan las imágenes $y$, en consecuencia, las funciones concretas que desarrolla la atenuación. De ahí que, aunque hablemos de macrofunciones, estas funciones tienen que perfilarse en relación con el género que particular que se esté analizando.

La autoprotección o autoimagen es la función centrada en el yo y se manifiesta cuando el hablante vela por los propios intereses, tratando de ganar o no perder la imagen. En el 
caso del género de las mesas redondas de expertos, esta macrofunción puede concretarse en buscar la aprobación del contenido científico propuesto (Albelda 2018), que es lo que sucede en el siguiente ejemplo.

(4)

VT: yo creo que la OMS ahí se equivoca// la obesidad es el resultado de muchas causas// no una enfermedad concreta

(Albelda 2018:358)

En este caso, el hablante atenúa la afirmación "la OMS ahí se equivoca" mediante la restricción de lo dicho a un punto de vista particular (el del hablante). De esta manera, hace más aceptable la crítica que plantea y se muestra como una persona que sigue las convenciones de la comunidad científica.

La prevención de una posible amenaza a la imagen o desacuerdo con interlocutor es una macrofunción que ayuda a propiciar el acuerdo con las propias ideas.

(5)

D: sí / eh con la venia/ eh este contenedor eh ha dicho usted que es difícil de mover y tal eh mmh está sobre unos RAílES que en esos raíles sí que hay unas [ruedas ((internas))]

J: [(( $\quad))]$ señor letrado// vamos a ver es que está usted sentando afirmaciones que no han sido manifestadas por el testigo entonces lo que no voy a dejar es que siente unas [premisas =]

T1: $\quad$ [mmh]

$\mathrm{J}:=$ para que en base a ellas conteste al testigo§

D: $\quad$ \$bien pueh- bueno

J: es que si no eeh está usted sentando la respuesta y lo que se trata es que la respuesta [y lo=]

D: [¿yo?]

$\mathrm{J}:=$ que se trata es que la respuesta nos la dé el testigo no el abogado§

$\mathrm{D:}$

J:

$\S y a \S$

§ivale?

D: vale/ bueno puees eh $\rightarrow$ este contenedor ¿de qué color es? ¿y de qué material está hecho?

Villalba (2017:74-74)

En este ejemplo, que pertenece a un juicio oral, el juez interrumpe para amonestar al letrado por introducir conocimiento personal en el interrogatorio. Los mecanismos que utiliza para mitigar la amenaza de este reproche y la prohibición de seguir por esa línea de interrogatorio son fundamentalmente las justificaciones y las marcas de control del contacto. Mediante estos recursos, busca que el interlocutor se muestre de acuerdo con su postura.

Por último, la función de reparación de un daño a la imagen del otro sirve para resolver los conflictos que puedan poner en peligro la obtención de una determinada meta discursiva. La diferencia fundamental entre esta función y la prevención es que para que exista reparación ya se debe haber producido una amenaza. Por lo que respecta a la meta discursiva, como en el ejemplo, puede resumirse en mantener la cordialidad con el resto de participantes.

(6)

D: si son toas unas putas/ hombre

A: no/ todas no§

D: $\quad \S$ ¿no?

A: mi madre no

D: clar- HOMBRE $\downarrow$ exceptuando (RISAS) a nuestras madres///

(Briz et al. 2002:55) 
Para otros autores, como Caffi (1999), es suficiente con distinguir únicamente dos grandes funciones: una orientada al propio hablante y otra orientada al otro, de modo que en este marco teórico prevención y reparación aparecerían unidas.

En cualquier caso, lo interesante de reconocer la función del atenuante reside en que constituye una herramienta para identificar la motivación que se esconde detrás del uso del elemento atenuante y valorar si tiene un uso estratégico o no.

\section{EL CONTEXTO INTERACTIVO: GENERAL Y CONCRETO}

La atenuación, en tanto fenómeno pragmático, requiere la evaluación del contexto para poder entender mejor su funcionamiento. El término contexto hace referencia al marco en el que se produce un enunciado y su definición es tan amplia que abarca desde el espacio físico y temporal hasta el conocimiento del mundo (y mutuo) de los participantes en la interacción. El contexto también integra valores y saberes culturales que, al igual que los ámbitos anteriormente señalados, inciden en la manera en que se produce la comunicación. Así pues, decir que el analista tiene que tener en cuenta el contexto para llevar a cabo un buen análisis de la atenuación significa que tiene que conocer muy bien las características del escenario y los atributos de los participantes que interactúan en la comunicación.

Meyer-Hermann (1988) insiste en la necesidad de conocer lo que él denomina contexto interactivo. Dentro de este contexto, es vital identificar las características del tipo interactivo, que este autor define a grandes rasgos como género discursivo. En la misma línea, Briz y Albelda (2013) dividen el concepto contexto en dos vertientes para el estudio de la atenuación: el contexto interactivo general y el contexto interactivo concreto.

Por una parte, el contexto interactivo general (CIG) hace referencia a las características del registro (relación de igualdad o jerarquía entre los participantes, saber vivencial compartido o no compartido, marco de la interacción cotidiano o no cotidiano, fin interpersonal o no interpersonal, mayor o menor grado de planificación) que entran en relación con las características y restricciones que plantea el género (Briz 2001).

En una conversación, que es el género que prototípicamente se asocia al registro coloquial, encontraremos generalmente una relación de igualdad entre los participantes, un saber vivencial compartido, un marco de la interacción cotidiano y un discurso poco planificado cuya finalidad es fundamentalmente interpersonal. Precisamente, esa finalidad interpersonal, junto con el carácter dialógico del género, propiciará la aparición de mecanismos de atenuación relacionados con la función de prevención o reparación (si es que hay algún conflicto).

En otros géneros, como los artículos de investigación, la atenuación se pone al servicio de cuidar la manera en que el propio autor se presenta ante el resto de la comunidad científica y predominará la función de autoimagen (Morales, 2010).

Por otro lado, el contexto interactivo concreto $(\mathrm{ClC})$ requiere de un análisis de la situación particular en la que aparece la forma candidata a desarrollar atenuación. Briz y Albelda (2013) proponen acotar este contexto a la identificación del elemento causante o desencadenante de la atenuación, al elemento o segmento atenuante (el recurso atenuante propiamente dicho) y al segmento atenuado. Si volvemos al ejemplo (6), el elemento desencadenante de la atenuación es la protesta de A "mi madre no" ante la descalificación 
de todas las mujeres. El elemento atenuador es la reformulación que lleva a cabo D y el segmento atenuado sería la intervención que había hecho antes el mismo hablante en referencia a las mujeres.

En definitiva, la integración del género en el estudio de la atenuación supone una ventaja para el analista que, al ser conocedor de las características que enmarcan la atenuación, tendrá más facilidad para reconocer los mecanismos que aparecen con más frecuencia así como las funciones concretas que realiza la atenuación en ese género en particular.

\section{OTRAS PRUEBAS DE ANÁLISIS: AUSENCIA, CONMUTACIÓN Y SOLIDARIDAD}

Las pruebas que se proponen a continuación han sido aplicadas a ejemplos de corpus de distintos géneros discursivos y han resultado ser útiles. Estas pruebas se han elaborado a partir de los propios análisis y de la reflexión sobre los ejemplos que analizan otros especialistas y que utilizan (a veces de manera intuitiva) para probar que se está ante un caso de atenuación.

\subsection{Prueba de ausencia}

La prueba de ausencia es, quizás, la más reveladora. Para aplicar esta prueba, es necesario eliminar el elemento atenuante y observar si se producen cambios en la fuerza ilocutiva.

Si la eliminación provoca que la fuerza ilocutiva se incremente o adquiera valores neutros, estaremos ante un elemento atenuante. Si, por el contrario, no se produce ningún cambio en la fuerza ilocutiva, se tratará de una forma no atenuante.

(7) a. I: Entonces eeh él quería para sus hijos y para sus nietos que fueran / unas personas eeh que no les faltara el cariño y de tanto que quería pues me parece que nos agobiaba un poco / pero / lo contaba él así con ese sentimiento que a veces eeh me acuerdo eeh / que lo he visto una vez llorar contando eso

b. I: de tanto que quería nos agobiaba

Corpus PRESEEA Valencia VALE_M33_010

En (7a) se han considerado como formas candidatas a desarrollar atenuación parecer y un poco. Tras aplicar la prueba de ausencia (7b) apreciamos que la aserción de I se vuelve más dura, el compromiso con lo dicho es mayor y, como consecuencia, la aserción es más directa. Existe, por tanto, una impacto en la fuerza ilocutiva.

A esta prueba se le pueden sumar los criterios que hemos mencionado en los epígrafes anteriores. Si atendemos al contexto interactivo general, por ejemplo, observamos que estamos ante un ejemplo extraído de una entrevista en la que se preguntan por aspectos personales al entrevistado. Dentro del contexto interactivo concreto, el elemento desencadenante de la atenuación es el verbo agobiar, que justifica la aparición de los mecanismos atenuantes anteriormente señalados: el primero para crear una distancia sobre el enunciado y acotar lo dicho al terreno de la percepción personal y el segundo para reducir la carga semántica negativa de agobiar.

Por lo que respecta a la función de las formas destacadas, se disminuye el contenido negativo de lo dicho sobre el abuelo del hablante para que este no proyecte una imagen de una persona que desprecia el cariño de su familia. Se trata, por tanto, de un ejemplo de autoprotección. 


\subsection{Prueba de conmutación}

En ocasiones no es posible eliminar el elemento lingüístico, por lo que hay que intentar sustituirlo por otras opciones que podrían aparecer en su lugar. Pensemos en un caso como el de (8a).

(8) (a) A: la pensión hay que reponérsela / señoría

Corpus Corpus Val.Es.Co. del Discurso Jurídico Oral [inédito] SVA 48

De acuerdo con lo que hemos visto, aquí hay varios indicadores que pueden hacernos pensar que estamos ante un enunciado atenuado. En primer lugar, observamos que este enunciado constituye un acto de habla directivo en el que se moviliza al interlocutor (el juez) para que lleve a cabo una acción (dicte una sentencia en la que se reconozca el derecho del demandante a continuar percibiendo una pensión). En segundo lugar, nuestro conocimiento del género (juicio oral) y del registro (formal) nos ofrece algunos rasgos para caracterizar el contexto interactivo general, como el hecho de que el juez ostente una posición jerárquica superior al hablante, que representa al demandante. En tercer lugar, dentro del contexto interactivo concreto, identificamos como desencadenante a un elemento no presente en la transcripción, pero sí en la mente del abogado: la necesidad de que la petición sea bien recibida por el juez y prevenir que la imagen de este pueda verse dañada. En cuarto lugar, identificamos como recurso de atenuación el uso de la perífrasis obligativa hay que. Como no podemos eliminar esta perífrasis, se sustituye por otros elementos que puedan desarrollar la misma función. En el caso de (8b) se ha optado por la versión en la que la fuerza ilocutiva se presenta de manera más directa.

(8b) Repóngale la pensión

Al contrastar (8a) y (8b) se aprecia que al hacer más visible a la persona que tiene que desarrollar la acción en (8b), el grado de obligación asciende y se altera, por tanto, la fuerza ilocutiva. Así pues, tenemos varios argumentos para pensar que la perífrasis actúa en este ejemplo como un mecanismo de atenuación.

\subsection{Prueba de solidaridad}

La atenuación no suele aparecer de manera aislada (Albelda et al. 2014:41), de modo que la presencia de más mecanismos atenuantes en el segmento que se esté analizando es un argumento a favor de que el elemento que nos plantea codifique atenuación. Pensemos en un ejemplo como el siguiente, extraído de una interacción médico-paciente:

(9) Dr. B: lo que se debe hacer es/ ir modificando un poco/ [el es-=]

A:

Dr. B: =el estilo dee/ de vida//

[nunca] tuvee $\rightarrow$

(Hernández Flores y Rodríguez Tembrás, 2018:332)

Tal y como explican las autoras, en este ejemplo encontramos varios mecanismos de atenuación: la construcción impersonal lo que se debe hacer, la perífrasis ir modificando y un poco. El contexto interactivo general, la consulta médica, es un espacio en el que existe una relación de jerarquía entre los participantes y en el que frecuentemente se realizan prescripciones y consejos para mejorar la salud de los pacientes. Es lo que sucede en este contexto concreto, donde el elemento desencadenante es, precisamente, la sugerencia de que se modifique del estilo de vida. El conflicto se genera porque se pone en cuestión la dieta de la paciente y esta siente amenazada su imagen. En consecuencia, para que las recomendaciones sean mejor recibidas, el médico trata de prevenir un posible rechazo 
reduciendo el grado de obligación de sus enunciados. La confluencia de una perífrasis obligativa construida con un mecanismo que desfocaliza al agente, el uso de una perífrasis incoativa que presenta el cambio como un proceso y el aproximativo un poco que también minimiza la intensidad del cambio que debe desarrollar la paciente nos hacen pensar que todos los mecanismos tienen una orientación atenuante.

Por último, cabe destacar que la prueba de la solidaridad es la menos robusta en comparación con las anteriores, porque tiene un valor orientativo. No obstante, no está de más tenerla en cuenta cuando nos encontremos ante casos dudosos.

\section{A MODO CONCLUSIONES}

En este trabajo se ha tratado de dar cuenta de varias herramientas que pueden servir de ayuda al analista en la identificación de elementos atenuantes. Contar con catálogos donde se recopilen recursos al servicio de la atenuación puede ser de gran utilidad; sin embargo, la identificación de la forma que desarrolla atenuación debe acompañarse de una reflexión que respalde el análisis. Si esto no sucede, se corre el riesgo de sesgar el análisis y caer en una lectura impresionista de los datos.

El primer criterio que se propone para identificar los casos de atenuación es observar una reducción de la fuerza ilocutiva. En relación con esto, la fuerza ilocutiva puede verse afectada directamente o puede alterarse mediante la expresión de vaguedad semántica o la desfocalización del papel de los participantes.

El segundo criterio que podemos emplear es la (macro)función que desarrolla la atenuación. La atenuación no deja de ser una estrategia argumentativa, como consecuencia, debe poder identificarse la motivación que lleva a su empleo. En caso contrario, lo más probable es que el elemento que se esté analizando no sea un mecanismo atenuante.

El tercer criterio está muy relacionado con la función y se deriva de la naturaleza pragmática del fenómeno. La atenuación es contextual, por lo que debe atenderse tanto al contexto interactivo general como al contexto interactivo concreto (Briz y Albelda, 2013). En esta línea, los últimos trabajos sobre el estudio de la atenuación están poniendo el foco en la manera en que el género puede condicionar el modo en que esta se manifiesta.

Finalmente, las pruebas propuestas (ausencia, conmutación y solidaridad) constituyen una ayuda complementaria para entender mejor la manera en que funcionan los criterios presentados por la bibliografía tanto en géneros escritos como orales. Sobre estos últimos, los trabajos más recientes ya empiezan a apuntar criterios prosódicos objetivos (Cabedo Nebot, 2018b) que, a medida que se vayan perfilando, supondrán un apoyo inestimable para el analista. 


\section{BIBLIOGRAFÍA}

AlbeldA, Marta (2010a): «¿Cómo se reconoce la atenuación? Una aproximación metodológica basada en el español peninsular hablado", en ORLETTI, Franca y Laura MARIOTTINI, eds., (Des)cortesía en español. Espacio teóricos y metodológicos para su estudio, Roma/Estocolmo: Università degli Studi Roma Tre-EDICE, 47-70.

AlbeLDA, Marta (2010b): «Influencia de los factores situacionales en la codificación e interpretación de la descortesía», Pragmatics, 18(4), 751-773.

AlbelDA, Marta (2016): «Sobre la incidencia de la imagen en la atenuación pragmática», Revista Internacional de Lingüística Iberoamericana (RILI), 27, 19-37.

AlbeLDA, Marta (2018): «La variación genéricodiscursiva de la atenuación como resultado de la variación de la imagen», Spanish in Context, 15(2), 346-368.

AlbeldA, Marta, Antonio Briz, Ana María Cestero, Dorota KotWICA, y Cristina VILLALBA (2014): «Ficha metodológica para el análisis pragmático de la atenuación en corpus discursivos del español. (ES.POR.ATENUACIÓN)», Oralia, 17, 7-62.

AlbeldA, Marta, y Ana María Cestero (2011): «De nuevo, sobre los procedimientos de atenuación lingüística», Español Actual: Revista de Español Vivo, 96, 9-40.

Albelda, Marta, y Ana María Cestero (2012): «La atenuación lingüística como fenómeno variable», en Cestero, Ana María, Isabel Molina y Florentino Paredes, eds., La lengua, lugar de encuentro. Actas XVI Congreso Internacional de la Alfal (Alcalá de Henares, 6-9 de junio de 2011), Universidad de Alcalá: Servicio de Publicaciones de la Universidad de Alcalá, 1857-1866.

BAZZANELlA, Carla (2002): "The Significance of Context in Comprehension: The 'WeCase'", Foundations of Science, 7(3), 239-254.

Bazzanella, Carla, Claudia CAFFI, y Marina SBISÀ (1991): "Scalar dimension of illocutionary force», en I. Zagar, ed., Speech acts: fiction or reality?, Liubliana, IPrA, 63-76.

BORETTI DE MACCHIA, Susana y María Cristina FERRER DE GREGoret (1984): «El español hablado en Rosario: diminutivos», Cuadernos de Literatura, 3, 93-116.

BRIZ, Antonio (1995): «La atenuación en la conversación coloquial. Una categoría pragmática», en Luis Cortés, ed., El español coloquial. Actas del I Simposio sobre análisis del discurso oral, Almería, Servicio de Publicaciones de la Universidad de Almería, 103-122.
Briz, Antonio (2001): El español coloquial en la conversación, Esbozo de pragmagramática, Barcelona, Ariel.

BRIZ, Antonio (2006): «Atenuación y cortesía verbal en la conversación coloquial: su tratamiento en la clase de ELE", Actas del programa de formación para profesorado de ELE, Munich, Instituto Cervantes, 227-255.

BRIZ, Antonio (2011): «La atenuación como categoría pragmática», Actas del VII Congreso Internacional de la Asociación Asiática de Hispanistas, Pekín, Foreign Language Teaching and Research Press.

BRIZ, Antonio (2012): «La (no)atenuación y la (des)cortesía, lo lingüístico y lo social: ¿son pareja?», en Escamilla Morales, Julio y Henry Vega, eds., Miradas multidisciplinares a los fenómenos de cortesía y descortesía en el mundo hispánico,Barranquilla/Estocolmo, Universidad de Estocolmo/Universidad del Atlántico/CADIS/Programa EDICE, 33-75.

Briz, Antonio, y AlbeldA, Marta (2013): «Una propuesta teórica y metodológica para el análisis de la atenuación lingüística en español y portugués. La base de un proyecto común (ES.POR.ATENUACIÓN)», Onomazéin, 28, 288-319.

CABEDO NeBOt, Adrián (2018a): «Creación de un modelo estadístico predictivo para la determinación de las funciones de atenuación en español hablado», Rilce. Revista de Filología Hispánica, 34.3, 1009-1027.

CABEDO NEBOT, Adrián (2018b): «Atenuación con disminución prosódica significativa en géneros con distinto grado de planifcación discursiva», Spanish in Context, 15(2), 218-236.

CAFFI, Claudia (1999): «On mitigation», Journal of Pragmatics, 31, 881-909.

CAFFI, Claudia (2007): Mitigation, Amsterdam, Elsevier.

FIGUERAS, Carolina (2018): «Atenuación, género discursivo e imagen», Spanish in Context, 15(2), 258-280.

HaverKATE, Henk (1992): «Deictic categories as mitigating devices», Pragmatics, 2, 505-522.

HeRnÁndeZ Flores, Nieves (2013): «Actividad de imagen: caracterización y tipología en la interacción comunicativa», Pragmática sociocultural, 1, 1-24.

HeRnÁndez Flores, Nieves y Vanesa, RodríGueZ TEMBRÁs (2018): “'Lo que se debe hacer es cambiar un poco el estilo de vida'. Estrategias de atenuación en el consejo médico», Spanish in Context, 15(2), 325-345. 
HOLMES, Janet (1984a): «Hedging your bets and sitting on the fence: Some evidence for hedges as support structures», Te Reo, 27, 47-62.

HoLmES, Janet (1984b): "Modifying illocutionary force", Journal of Pragmatics, 8(3), 345-365.

HOLMLANDER, Disa (2006): «Atenuación con y sin cortesía. Un estudio de conversaciones interculturales entre españoles y suecos», en Briz Gómez, Antonio, Antonio Hidalgo Navarro, Marta Albelda Marco, Nieves Hernández Flores y Josefa CONTRERAS, eds., Actas del III Coloquio Internacional del programa EDICE, Valencia, Universitat de València, 730-754.

HYLAND, Ken (1996): «Writing without conviction? Hedging in science research articles», Applied Linguistics, 17, 433-454.

LAKOFF, George (1973): «Hedges: A Study in Meaning Criteria and the Logic of Fuzzy Concepts», Journal of Philosophical Logic, 2, 458-507.

LYSVÅG, Per (1975): "Verbs of hedging», en P. KIMBALL, John, ed., Syntax and Semantics, Vol. 4, Nueva York, Academic Press, 125-154.

MeYer-HeRMAnN, Reinhard (1988): «Atenuación e intensificación (análisis pragmático de sus formas y funciones en el español hablado)", Anuario de Estudios Filológicos, 11, 275-290.

MIHATSCH, Wiltrud (2018): «De la escritura científica a la conversación coloquial adolescente. E caso de "tipo"», Spanish in Context, 15(2), 281304.

MoRALES, Óscar A. (2010): Los géneros escritos de la Odontología hispanoamericana. Estructura retórica y estrategias de atenuación en artículos de investigación, casos clínicos y artículos de investigación, Barcelona, Pompeu Fabra.

SBISÀ, Marina (2001): «lllocutionary force and degrees of strength in language use», Journal of Pragmatics, 33, 1791-1814.

SCHNEIDER, Stefan (2013): «La atenuación gramatical y léxica», Oralia, 16, 335-356.

THALER, Verena (2012): «Mitigation as modification of illocutionary force», Journal of Pragmatics, 44, 907-919.

VILLALBA, Cristina (2016): Actividades de imagen, atenuación e impersonalidad en los juicios orales, Universitat de València, Valencia.

VILLALBA, Cristina (2017): Actividades de imagen, atenuación e impersonalidad. Un estudio a partir de juicios orales españoles, Frankfurt am Main, Peter Lang.

VILLALBA, Cristina (2018): «Primera persona del plural en los juicios orales. Valor representativo y estrategia atenuante», Revista del Instituto de Lengua y Cultura Españolas (RILCE), 34(3), 1056-1080.

ZADEH, Lofti Asker (1965): «Fuzzy sets», Information and Control, 8(3), 338-353. 